We present the dramatic course of a female 5-year-old child with pneumonia and symptoms similar to local compression of the pericardium by a tumorous mass originating from the left lung. The child was treated with antibiotics for pneumonia with bilateral pleural effusions that required chest drainage. On the 10th day of therapy there was sudden anemia observed with the echocardiographic finding of acute cardiac tamponade. The child was referred for emergency life-saving surgical intervention. The chest was opened via a minimally invasive mini-incision in the area of the xiphoid process and bloody tension pericardial effusion was evacuated. The laboratory and histopathology investigations were not specific for neoplastic disease or tuberculosis infection. In the further observation the girl recovered and was discharged home two weeks after tamponade drainage. Fortunately our initial suspicion of neoplastic disease was not proved; nevertheless we would like to emphasize the need for oncologic vigilance in similar cases.

Key words: acute tamponade, lung tumor, cardiac surgery, viral pneumonia.

\section{Bloody pericardial tamponade in a child treated for pneumonia mimicking a lung tumor and infiltration of the heart}

Ireneusz Haponiuk ${ }^{1}$, Maciej Chojnicki ${ }^{1}$, Radoslaw Jaworski', Jacek Zielinski ${ }^{2}$, Ninela Irga-Jaworska ${ }^{3}$, Katarzyna Gierat-Haponiuk ${ }^{4}$, Mariusz Sroka ${ }^{5}$

1Department of Pediatric Cardiac Surgery, Mikołaj Kopernik Pomeranian Centre of Traumatology, Gdansk, Poland

2Department of Surgical Oncology, Medical University of Gdansk, Poland

${ }^{3}$ Department of Pediatrics, Hematology, Oncology and Endocrinology,

Medical University of Gdansk, Poland

${ }^{4}$ Department of Rehabilitation, Medical University of Gdansk, Poland

${ }^{5}$ Department of Pediatric Surgery and Urology for Children and Adolescents,

Medical University of Gdansk, Poland

\section{Introduction}

Acute tamponade especially with bloody fluid should always require oncological diagnostics of potential neoplastic growth [1, 2]. There are only a few reports of acute bloody pericardial tamponade in immunocompetent patients due to viral infection [3]. We present the dramatic course of a female 5-yearold child with pneumonia and symptoms similar to local compression of the pericardium by a tumor-mimicking mass originating from the left lung [3].

\section{Case report}

A five-year-old child, body weight $23 \mathrm{~kg}$, with acute cardiac tamponade in the course of treated pneumonia, was referred from the local hospital for emergency surgical intervention. The reason for hospitalization was abdominal pain with nausea; C-reactive protein was in the normal laboratory range $(<5.0 \mathrm{mg} / \mathrm{l})$; no changes in chest $X$-ray were present. Four days after admission pneumonia of undefined etiology was suspected. The patient underwent antibiotic treatment (amoxicillin + clavulanic acid $3 \times 480 \mathrm{mg}$ i.v. for 6 days, netilmicin $3 \times 40$ mg i.v. for 4 days), and the imaging diagnostics suggested left lung pneumonia with left inferior lobe atelectasis, which impressed the pericardium in a way similar to a left lung tumor with aggressive local growth. The laboratory investigations were not specific for neoplastic disease or tuberculosis infection, although there were atypical forms in the blood shape.

The child was initially referred for chest drainage because of the left pleural effusion (Fig. 1), but the chest drainage did not improve severe hypoxia caused by dyspnea at rest and shortness of breath, despite constant oxygen delivery. After the next 2 days, despite pleural drainage and change of antibiotics (ceftazidime $2 \times 500 \mathrm{mg}$ i.v. and fluconazole $1 \times 50 \mathrm{mg}$ i.v.), the child deteriorated rapidly due to rapid symptoms of heart failure, shortness of breath and fatigue. In the echocardiography performed just before the intervention there was acute tamponade with local impression of the left atrium and the left ventricle, with functional mitral valve regurgitation grade II (MVI++), without any congenital defects (Fig. 2). The child was referred for an emergency operation aimed at pericardial tamponade evacuation.

Surgical intervention was performed with the cooperation of a multidisciplinary team (cardiac surgeon, pediatric surgeon, cardiologist, radiologist, oncol- 


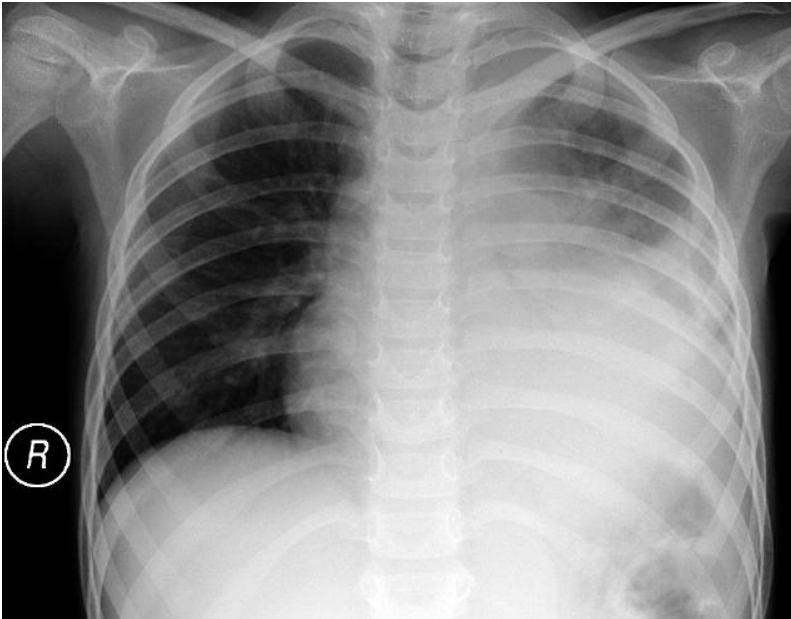

Fig. 1. Preoperative chest X-ray showing massive pleural fluid

ogist). The chest was opened via a subxiphoid minimally invasive median minithoracotomy incision. After meticulous dissection the pericardial sac was opened and bloody tension fluid was observed. The bloody effusion of $200 \mathrm{ml}$ was evacuated with dynamic outflow under pressure and the heart was dissected free from the fresh fibrous adhesions. In the direct inspection there was clear evidence of solid extrapericardial impression in the area of the left atrium; therefore all manipulation in the pericardium was very gentle. The chest tube with active suction was left in the pericardial sac underneath the inferior wall of the heart. The specimens from peri-
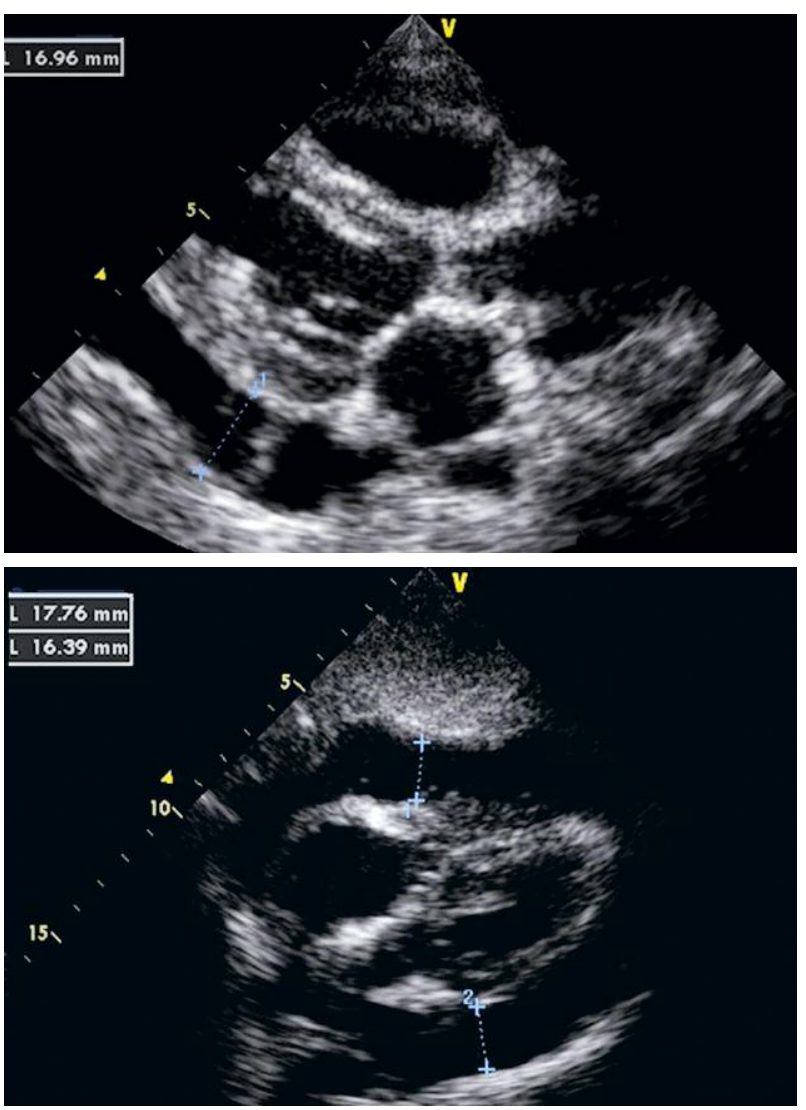

Fig. 2. Preoperative echocardiography showing acute tamponade with local heart impression cardial tissue and the fluid were harvested for further laboratory and histopathological tests. The girl was extubated directly in the operative room postoperatively. Postoperative computed tomography did not confirm pericardial infiltration and showed no clear evidence of mediastinal tumor (Fig. 3). The condition of the girl improved and after control echocardiography the drainage was removed 24 hours after surgery. The early postoperative period was uncomplicated. In the postoperative echocardiography there was persistent impression on the left ventricle with pericardial fluid separation inside the pericardium in the field of the left ventricle. Only after the surgery was completed were the previous blood cultures found to be negative. C-reactive protein was $50 \mathrm{mg} / \mathrm{l}$. Pericardial fluid examination revealed bloody liquid with the density of $1.01 \mathrm{~g} / \mathrm{l}(\mathrm{pH} 8$; protein $53.5 \mathrm{~g} / \mathrm{l}$; glucose 62 mg\%; LDH 1326 U/I; amylase 36 U/I). Histopathological examination of pericardium and pericardial fluid revealed no signs of neoplastic growth or atypical cells, with inflammatory reaction signs. Viral infection with no symptoms of malignant cells was finally suspected. The child was transferred back to the referring department of pediatrics for continuation of antibiotics on the $4^{\text {th }}$ postoperative day (ceftazidime, fluconazole). Control echocardiography showed no impression over the left ventricle and a small amount of fluid in the area of the left ventricle. The follow-up observation has now reached four years and there have been neither cardiac episodes nor pericardial fluid recurrence.

\section{Discussion}

The presented case is unique because of the age of the child and aggressive growth of the pericardial effusion leading to ongoing circulatory and respiratory compromise that suggested neoplastic tumor, but neither imaging studies nor histopathological examinations confirmed disseminative disease. An alternative to the diagnosis of tumor was acute viral infection with an undefined microorganism, with rapid disease course as well as pleural and pericardial fluids. There are similar reports of young children with rapid pericardial tamponade. Most of them are patients with circulatory insufficiency due to heart failure with mediastinal neoplasms and local impression of the heart [5].

In our case the decision to perform an emergency ministernotomy was undertaken in the face of a circulatory insuf-

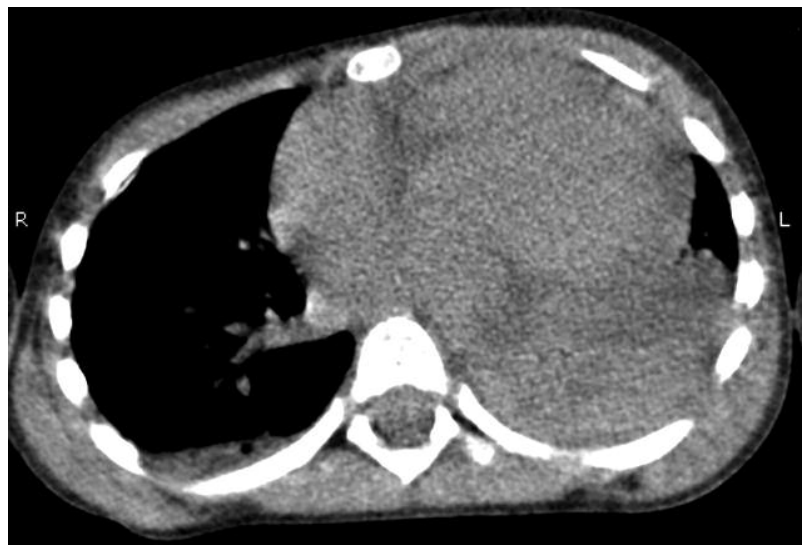

Fig. 3. Thoracic computed tomography image showing pleural fluid with left lung compression 
ficiency with awareness of a lack of final diagnosis in a patient in an emergency setting. The primary clinical diagnosis, confirmed by imaging, did not exclude a heart tumor, pulmonary neoplasm, or cardiomyopathy. We performed the procedure with the very best oncologic vigilance in emergency settings, despite the lack of a final diagnosis.

The presented minimally invasive approach proves the safe possibility of mediastinal exploration with effective fluid evacuation in emergency, which is our commonly used, and recommended in many centers, ministernotomy of choice. This method, although commonly reported as the second step after initial pericardial puncture, enables pericardial inspection, dissection of pericardial adhesions, and macroscopic evaluation of the heart, while being related to lower risk of complications [4]. Emergency tamponade drainage was necessary to save and stabilize the patient, who was subjected to continued antibiotic chemotherapy and further diagnostics. The clinical image observed while on perioperative treatment was unclear, more similar to an intrathoracic tumor than pneumonia with left lung atelectatic lobe. The presented clinical course can be a challenge for diagnostics and therapy that needs meticulous clinical examinations to prevent the oversight of potentially hazardous symptoms and terminal diseases, despite oncologic vigilance.

The good early result enabled early extubation and simultaneous to planned anesthesia weaning of the patient from mechanical ventilation, which corresponds to contemporary literature reports [1-6]. The procedure was the turning point for continuation of therapy, as well as for precise histopathological diagnosis and subsequent modification of further examinations and strategy of care [2].

The presented case report emphasizes the role of a multidisciplinary approach in critically ill children with pericardial tamponade. Acute tamponade especially with bloody fluid always requires oncological diagnostics of potential neoplastic growth. Although the rapid disease course was due to viral infection, we could not exclude AH1N1 etiology of infection; no such test was done in our department before the AH1N1 pandemic in 2009 [7, 8].

The authors declare no conflict of interest.

\section{References}

1. Martino F, Avila LF, Encinas JL, Luis AL, Olivares P, Lassaletta L, Nistal M, Tovar JA. Teratomas of the neck and mediastinum in children. Pediatr Surg Int 2006; 22: 627-34.

2. Priola AM, Priola SM, Cardinale L, Cataldi A, Fava C. The anterior mediastinum: diseases. Radiol Med. (Torino) 2006; 111: 312-42.

3. Kabukcu M, Demircioglu F, Yanik E, Basarici I, Ersel F. Pericardial tamponade and large pericardial effusions: causal factors and efficacy of percutaneous catheter drainage in 50 patients. Tex Heart Inst J 2004; 31: 398-403.

4. Zisis C, Rontogianni D, Stratakos G, Voutetakis K, Skevis K, Argiriou M, Bellenis I. Teratoma occupying the left hemithorax. World J Surg Oncol 2005; 22: 76.

5. Koga H, Yamataka A, Kobayashi H, Miyamoto H, Lane GJ, Miyano T. Median sternotomy provides excellent exposure for excising mediastinal tumors in children. Pediatr Surg Int 2005; 21: 864-7.

6. Haponiuk I, Rzyman W, Sroka M, Drożyńska E, Chojnicki M, Murawski M, Hinc-Kasprzyk J, Czauderna P. Malignant mediastinal and left lung tumor - macroscopically radical resection in an infant with a critical respiratory failure, report of a case. Kardiochirur Torakochir Pol 2010; 7: 31-3.

7. Koranyi K, Yontz D, Rohrer Z, Leber A, Ramilo O. Pericardial effusion complicating novel influenza A (H1N1) infection in an infant. Pediatr Infect Dis J 2010; 29: 782-3.

8. Irga N, Osak M, Jaworski R, Bronk M, Kosiak W, Adamkiewicz-Drozynska E. Pandemic (H1N1) 2009 influenza - real threat or unjustified panic? The experience of one pediatric hematology-oncology center. Centr Eur J Med 2012; 7: 296-301.

\section{Address for correspondence}

Ireneusz Haponiuk MD, PhD

Department of Pediatric Cardiac Surgery

Mikołaj Kopernik Pomeranian Centre of Traumatology

1-6 Nowe Ogrody Street

80-806 Gdańsk, Poland

tel. +48583220851

e-mail: ireneusz_haponiuk@poczta.onet.pl

Submitted: 26.01 .2012

Accepted: 18.02 .2013 